\title{
Exploring the health visiting service from the view of South Asian clients in
}

England: a grounded theory study

*Lena Abdu (RN, RHV, LLB, MSc), First Community Health \& Care, $2^{\text {nd }}$ Floor, Forum House, 41-51, Brighton Road, Surrey RH16YS

Lena.abdu@firstcommunitysurrey-cic.nhs.uk

Tel: 01737775479

Karen Stenner (PhD, BSc), Lecturer in Health Services Research, School of Health Sciences, University of Surrey

Vasso Vydelingum (PhD, BSc, PG Dip.Ed) Associate Senior Lecturer, School of Health Sciences, University of Surrey

*Corresponding author 


\begin{abstract}
The fact that health inequalities disproportionately affect the minority ethnic population is not new and projections are that the minority ethnic population will continue to increase. The importance of early intervention and the key role that health visitors can play in attempting to reduce health inequalities is well documented as is the requirement for health providers to establish culturally sensitive services. To date much of the research has focused on the perspectives of health care professionals caring for minority ethnic clients in hospital based settings and very little is known about the perspectives of minority ethnic clients regarding the health visiting service. The aim of this study was to explore the perspectives of South Asians regarding their experiences with the health visiting service. The study was conducted in a small town in the South of England between March and June 2013. A qualitative approach was selected using a grounded theory approach to capture the perspectives of this group regarding their interactions with the health visiting service. The sample consisted of 15 participants and data was collected through audio-recorded semi-structured interviews and analysed using constant comparative approach. Three key categories were identified: 'the journey to understanding the health visitor's role', 'the relevance of the service' for the participants and 'the significance of family'. It was found that South Asian clients distinguish between health and parenting advice, being more likely to accept health advice from their health visitor and more likely to accept parenting advice from their family. The findings, although limited in their generalizability, offer important insights into how South Asians perceive the service and will equip health visitors with a better understanding of how best to improve the experience of South Asian clients accessing the health visiting.
\end{abstract}

Keywords: Health visitor, minority ethnic clients, qualitative, South Asian, cultural sensitivity, public health nursing

\title{
What is already known?
}

- Health inequalities are rising and disproportionately affect minority ethnic clients

- There is a clear obligation on NHS trusts to reduce health inequalities

- Little is known about the perspectives of minority ethnic clients regarding the health visiting service 


\section{What this paper adds?}

- One-to-one support from a health visitor is valued by South Asian clients, particularly when suffering emotional distress

- South Asian clients who live with extended family can experience tension between the advice given by health visitors and that provided by the family.

- There are indications that South Asian clients are likely to accept health advice from their health visitor but are more likely to accept parenting advice from their family.

\section{Introduction}

Adapting and responding to the changing needs of an increasingly multi-cultural society is a challenge faced by those providing healthcare in the United Kingdom (UK) as in other developed countries. In the latest UK Census (Office for National Statistics 2013), white British had decreased from $87 \%$ in 2001 to $80 \%$ by 2011, and remaining ethnic groups had increased, giving health care workers caseloads with greater diversity than ever before. Health services in the UK have legal responsibilities to uphold the rights of individuals to healthcare that meets their needs, without prejudice, and to tackle inequalities (Human Rights Act 1998, Equality Act 2010). Health inequalities, defined as preventable and unjust differences in health status that impact on health in terms of shortened life expectancy and quality of life, disproportionately affect the minority ethnic population (Bhopal 2007, Liu et al. 2012).

In England, the health visiting service (HVS) is a universal service providing all new parents with a named health visitor whose role is to assess the health needs of families, support new parents, and tackle issues such as child protection, social isolation and domestic violence. Broadly, it aims to improve the health outcomes of children and their families and to tackle the impact of health and social inequalities through working closely with at-risk or deprived groups and in partnership with other specialist services (Department of Health (DH) 2011). Expansion of the service is currently underway through the recruitment and training of an additional 4,200 health visitors under the Coalition Government's policy, Health Visitor Implementation Plan 2011-2015 (DH 2011). This was a direct response to Marmot's Review (DH 2010) which highlighted the importance of early intervention in attempting to reduce the cycle of deprivation and the key role that health visitors can play in family centred 
interventions and community based public health. To qualify, registered nurses or midwives undertake an approved degree level programme typically lasting 1 year full time (www.nhscareers.nhs.uk).

UK policy emphasises the importance of assessing the impact of services on vulnerable groups, such as the minority ethnic population (DH 2007). There is therefore a need to explore the perspectives of minority ethnic clients to understand whether the health visiting service is meeting their needs.

\section{Background}

Cultural sensitivity in healthcare refers to respect for diversity and an awareness of how one's own culture, background and values may influence care delivery (Starr \& Wallace 2011). There is a lack of agreement over how best to improve cultural sensitivity as 'having cultural knowledge is necessary but not sufficient for the provision of culturally competent care' (Engebretson et al. 2008, p.176). Cultural competence requires a combination of appropriate skills, cultural knowledge and awareness (Suurmond et al. 2010). However, there is a need to recognise and expect diversity within, as well as between, ethnic groups in order to prevent unhelpful stereotyping (Engebretson et al. 2008). Models of cultural competency have been criticised for oversimplifying cultural differences and for focusing on practitioner-client relationships to the exclusion of political, structural and systemic practices that may regulate this behaviour and influence inequalities (Culley 2006, Green et al. 2006). In contrast, too heavy a focus on identifying organisational practices that perpetuate institutional racism within health services is criticised for failing to account for individual agency (Bradby 2010). These arguments illustrate a lack of clarity over how cultural competence operates at the individual and organisational level, raising questions about how best to improve clinical practice.

A range of factors is known to contribute to inequitable health care for ethnic minorities. Ability to access healthcare can be adversely influenced by language barriers, location and family choice, (Douglas 2012, Green et al. 2006). In relation to provider characteristics, inadequate communication, lack of involvement of clients in decision-making and negative or inappropriate interpersonal styles have been identified as key contributors to experiences of disparity in healthcare for minority ethnic clients (Starr \& Wallace 2011). A survey of health 
visitors $(\mathrm{n}=75)$ in England revealed a tendency towards an ethnocentric approach (Knight Jackson 2007), and that some health visitors lacked the skills required to assess and treat people from minority ethnic groups effectively (Donetto et al. 2013). Similar findings in other settings question the cultural sensitivity of healthcare workers (Vydelingum 2000, Leishman 2004, Garrett et al. 2008). Spence's (2001) study of nurses' experiences of nursing people from cultures other than their own in New Zealand highlight the tensions faced by nurses when encountering cultural differences and their struggles to deliver culturally appropriate care when faced with uncertainties and difference.

Given that the minority ethnic population of the UK is predicted to rise to twenty percent by 2051 (Wohland et al. 2010), there is a need for more research in this area. The health visiting workforce is expanding (DH 2011) and there is a requirement for health providers to collaborate with service users to define and establish culturally sensitive services (DH 2007:4).

This paper reports on a study which aims to explore the perspectives of South Asian clients regarding the health visiting service. It was envisaged that a study would illuminate the extent to which the health visiting service is meeting the clients health needs in order to inform future practice and the design of health visiting services regarding minority ethnic clients.

\section{Method}

\section{Design}

A qualitative interview study was undertaken as part of post graduate study by the principal researcher who was working as a health visitor. A classic grounded theory approach was chosen, Glaser (2002), in designing analysing and interpreting the data because of its emphasis on allowing emergent theory from the participant's perspective. It was felt that the inductive approach of grounded theory was best suited to address the aims of the study because of the lack of research on minority ethnic client's perspectives regarding the health visiting service.

\section{Participants}

Whilst aware of the criticism that the term South Asian may overemphasise the homogeneity of the group (Bradby 2003), the term South Asian has been employed in this small scale 
study to describe a person whose ancestry is in the countries of the Indian subcontinent, including India, Pakistan, Bangladesh and Sri Lanka (Bhopal 2004).

Participants were identified from the caseloads of health visitors and from weekly health visitor clinics within 1 geographical area in the South of England. Recruitment from the researcher's own case load was avoided to reduce potential influence of a prior relationship or coercion.

Potential participants were given a participant information leaflet and then telephoned within 2 weeks by the researcher to see if they wished to take part in the study. The recruitment criteria included participants who: (1) were parents between eighteen and sixty years, of South Asian origin, (born in the United Kingdom or overseas), (2) had an interaction with the health visiting service within the last two years, (3) could speak conversational English, (4) had a child not less than eight weeks of age and (5) agreed to participate.

\section{Data collection}

Face to face semi-structured interviews were undertaken between March and June 2012 by the first author. An interview prompt outlining themes was designed which included understanding of the health visitor's role, relevance of information given to their cultural background, the level of support given by the health visitor and any effect this had on parenting as well as overall impressions of how helpful the service had been. Interviews lasted between 45 to 60 minutes and were audio recorded and transcribed verbatim. A reflective diary was written immediately following each interview.

\section{Data analysis}

The data was managed adopting the approach of Strauss and Corbin (1998) which employs simultaneous collection, coding and analysis of data. Transcription began the data analysis process and promoted early immersion in the data, followed by hand labelling texts using a system of open, axial and selective coding. Constant comparison was used as a way of maintaining a connection between the codes and a constant re-examining of the text to ensure consistency of the application of that particular code and to ensure that codes and categories developed to fit the data. Analysis included search for deviant cases but none were found. A modified version of theoretical sampling was used whereby emerging categories from early data was used to refine questioning in subsequent interviews. The two co-authors then 
checked the coding and emerging categories independently for consistency and the process of analysis was reviewed and discussed together with the first author.

\section{Ethical considerations}

Ethical approval for the study was obtained through the NHS local ethics committee and the University of Surrey with local research governance obtained for the appropriate NHS site. Written consent was obtained by the first author and the researcher was equipped to refer to appropriate professional community based services who could provide psychological support if required.

\section{Rigour}

Rigour was enhanced by the two co-authors independently checking the subsequent fit between the emerging categories and theoretical understanding for consistency (Beck 1993). In keeping with grounded theory methodology, interview questions were adapted as concepts emerged and participants' meanings validated against emergent concepts, (Chivotti and Piran 2003). With an interest in delivering healthcare to minority ethnic groups, the researcher attended an ethnic minority parent and child playgroup prior to the study to increase awareness of existing specialist services and the needs of the local population. The researcher kept a reflexive diary to heighten awareness of blurring between professional and research roles.

\section{Findings}

A sample of 15 female participants was recruited aged between 20 and 37 years, see table 1 for more detail of participant characteristics. Data analysis resulted in three categories: 'the journey to understanding the health visitor's role', 'how relevant is this for me?' and 'the significance of family'. Categories are illustrated by anonymised quotations.

\section{Understanding the role of the health visitor}

Not understanding the role of the health visitor was the starting position that participants $(n=14)$ described when they first met the health visitor. Participants expressed feelings of being unsure of how and when to access the service, and uncertainty regarding the role of health visitors in relation to other healthcare professionals and their level of training. A reason articulated by more than half of the participants for their lack of understanding was that there was no comparable service in their homeland. 
"There's no set up like this in my country. What should I ask? It seems the health visitor wants you to ask questions" (PP6)

This uncertainty continued even after their initial meeting with the health visitor, with participants feeling unable to evaluate whether they had received the full service or not.

"I don't know what their job is. So I cannot say if something has been left out or not because I don't completely know what she is supposed to do...." (PP7)

Without clarity regarding the health visitor's role, participants were left to navigate their way in a 'sea of confusion.' The system was described as confusing and difficulties were reported in identifying the most appropriate service to use.

"For a long time I was looking for someone to check her weight. I don't know where I should go. Then I take her to the GP and they said the baby weight checking day is Wednesday..... At the clinic, other than weighing I don't know what other services they do..." (PP6)

Participants also felt reprimanded when, they tried to access the system at the 'wrong' juncture:

"I think it's complicated to know who to call.... There was this kind of blur as to where does this end and where do I go next, it's so complicated and they make you feel it's obvious who you should call. It might be obvious to them, but if I knew who to call or where to go I wouldn't have gone to the wrong place would I? " (PP5)

Despite the uncertainty over what the health visiting service could provide, nearly all participants expressed confidence and trust in the system.

"I was just going to wait and see what they were there for. I trust the system here so I thought everyone has their job to do in this process at a particular time" (PP5)

Although participants began to gain insight into the service if and as their exposure increased, there was a sense that they remained on the 'back foot' throughout their trajectory with the HVS.

\section{Sensitivity of services}


This category explores the relevance and cultural sensitivity of different aspects of the health visiting service and the impact on participants in relation to language, cultural differences and relationships. Findings suggest that explanations given about the health visiting service were not meaningful to all participants.

'Lost in translation' describes the situation whereby an original meaning cannot be translated perfectly word for word into another language and needs to be rephrased into something the recipient can make sense of. Despite speaking conversational English, half of all participants seemed to lack confidence in questioning the health visitor, and seeking out more meaningful explanations:

“They wrote his weight down in the red book. If they didn't say anything I just thought everything must be OK" (PP7)

In addition, several participants mentioned that they avoided telephone contact because of concerns that people would not understand their accent or that they might not be able to understand the health visitor.

"I didn't understand everything she said. She spoke quite fast and I didn't want to make her late for her next visit ..." (PP3)

Cultural challenges were reported, including differences in what is considered gender appropriate conversation and different cultural style encountered from the health visiting service compared to that of their homeland. Cultural differences also extended to the delivery of the service which, other than clinics, used appointment systems for home visits or health visiting led courses which a client must book onto in advance:

"It's just all more formal here. You have an appointment and then the health visitor who you've never met comes to your home and asks you so many things....... Back home it's simple; here there are a lot more rules..." (PP2)

The majority of participants confirmed that they had not attended any health visiting led group and indicated that lack of familiarity with attending groups was a factor in their decision not to attend. It was also found that advice given was not always attuned to the participant's cultural stance and the lifestyle changes they might experience as they adjusted to living in the UK: 
"The first time I met her she said a bit about going out for walks and joining things. Traditionally in India you wouldn't go out for the first 14 days at least. She said I should take the baby out for fresh air and that lots of mothers are out and about by now. I told her that in India you would probably just keep yourself covered and stay indoors as much as possible. If I tell my mother in law I'm going out for a walk with a two week old baby in March she will think I have gone crazy. I don't think the health visitor wanted to hear that" (PP9)

Half of all participants felt that the health visitor gave priority to standardised advice, leaving participants feeling that their own customs were considered incorrect.

"Sometimes she makes me feels as if I'm not doing it right... I feel as if she doesn't approve of the way I'm doing things... I get the feeling that they want everything done a certain way" (PP11)

Throughout the study participants expressed feelings of 'a disconnect' between what they knew from their homeland and what they were now experiencing, yet two thirds of participants described the contact they had with a health visitor as being helpful. While some participants expressed reluctance to allow a 'stranger' into their home, relationships with health visitors were valued once they had developed and this increased their readiness to engage with the service.

"Now that I know I can ask when she will be in clinic I would prefer to go and see her before just calling the GP" (PP5)

This relationship was most valued by participants who found themselves without their extended family nearby:

"I did feel she understood I was alone here. With the first baby I got depression... and the good thing is she came and taught me baby massage.... and we used the coconut oil from back home. I remember that because normally we use it for the girls' hair but the health visitor said it was fine as long as he didn't have any allergy to it. I remember I felt a lot better after those visits" (PP4)

This participant reveals the powerful impact of being understood by a health visitor during her period of postnatal depression and being able to bring her two worlds together, using coconut oil from her homeland to massage her baby in her current home. 


\section{The significance of family}

Participants attached great value to the involvement of their family in childrearing, whether the extended family lived in the UK or in their homeland. This was reflected in the common use of the term 'we' referring to the family, as opposed to ' $I$ ' being the mother. All participants had expectations of their family to help them when they had a baby, and that this was shaped by the family structure and their experiences of their homeland:

"Back home it's just family, mothers and aunties helping you, and it would be a shame on them if they didn't help you" (PP3)

It was noted that participants referred to home as the place where their extended family lived and that none mentioned a network of local friends. This reliance on family as a social network was at odds with health visitor goals to encourage new parents to attend health visiting led groups.

"I don't know what you have to do when you get there. Is it something like a talk? ... I don't tend to go out for things like that, not on my own..... at the weekends my husband will take us out so that we don't need to worry about going out in the week..." (PP7)

In contrast, for those participants whose extended family lived overseas, the health visitor role was described as important for providing support and reducing isolation and loneliness.

"I never imagined that I would be on my own all day when I had my baby. Back home there is always someone to help you, aunties, cousins even neighbours...Here it's really hard, completely different, everyone keeps to themselves, so just having the health visitor visit for an hour makes a difference otherwise I might go the whole day without talking to someone until my husband got home" (PP4)

There was general agreement that the health visiting service did not fully understand the significance of family involvement in childrearing the South Asian context. Those living with their extended families unanimously used the phrase "they don't understand" or "misunderstand" when referring to the health visitor.

"They don't understand that in our culture a baby is part of a whole family and everyone helps out, not just the mother" (PP8) 
There was a feeling that health visitors preferred to focus attention on the mother alone and sometimes made incorrect assumptions that the extended family would interfere.

"It was almost like the health visitor decided as soon as she saw them [the family] that they would be interfering? And I was just like 'No I don't find them interfering, in fact it really helps me that they're here'.'

As the family played a significant role in providing and/or making decisions about childcare, the majority of participants reported instances where they had to manage conflicting advice from the extended family and the health visitor. Conflict often featured over what is acceptable to feed a baby, with several Hindu participants mentioning their mothers wish to give the baby a taste of honey or almond paste which is contrary to current health visiting advice based on Department of Health recommendations:

“Back home it's very common to put a little taste of honey on the baby's tongue.

Fortunately my in laws haven't pushed it ...or maybe they just did it when I wasn't looking?" (PP10)

Some participants reported feelings of stress as they tried to reconcile two contrasting cultural models of parenting, not wishing to displease family members. Extended families did however appear to be more accepting of advice that was health related, for example, concerning vitamins, immunisations and attending developmental checks, rather than parenting advice which was considered to be their domain.

"I mean from a health point of view the family like the advice but some things, like maybe how to raise a kid, they will listen but I know they will do their own thing, that's the way they know.." (PP15).

\section{Discussion}

Overall, while these South Asian clients expressed few direct concerns, there were difficulties and barriers that disrupted their journey through the HVS.

It is widely acknowledged that language can be a barrier for ethnic minorities in accessing health care (Henderson 2011, Yu 2011). While able to speak English, lack of confidence in its use was a deterrent to accessing and understanding services for some participants. Similar barriers have been reported among Chinese migrant workers, including those fluent in English (Green et al. 2006). As participants in this study spoke conversational English, 
translation services would not have been offered, however research suggests these services are not universally offered when required, and where provided such translation services have been found to be problematic (Alexander et al. 2004).

With little previous knowledge of HVS, a major disadvantage was in not knowing what to expect and how to navigate NHS systems. Difficulty in understanding how health systems work, and when and how to access services, are also barriers for asylum seekers in the UK (O'Donell et al. 2007). While these difficulties are not specific to ethnic minorities (for example, lack of knowledge about HVS is common amongst first time parents (Donetto et al. 2013)), confusion remained over time for some participants, indicating continuing communication barriers. Donetto et al. (2013) argue that an understanding of how best to use the HVS is gained through a process of reciprocal exchange and the development of trust between provider and client. This can be achieved through a range of attitudes, skills and activities in what Cowley et al. (2013) identify as a health visitor 'orientation to practice'.

Ineffective provider communication is considered a key reason that minority ethnic clients experience health disparities and consequently are unable to fully participate in decision making (Garrett et al. 2008, Starr \& Wallace 2011). This study identified instances of power in-balance whereby clients wanted to question the health visitor and check their understanding but perceived this to be inappropriate. Advice given was sometimes at odds with cultural values and health visitors were seen as reluctant to accept challenges to this advice. This is reminiscent of ethnocentric and monocultural approaches to health described in previous studies, (Vydelingum 2006, Knight Jackson 2007). As with previous research (Donetto et al. 2013), negative experiences in past encounters with health professionals had left a lasting impression on participants, however where HVs were flexible and client led, such an approach was found to be both effective and empowering for the client.

Services were not always perceived to be aligned to participants' cultural values and beliefs around, for example, sensitivity about revealing personal matters to strangers and involving men in women's health issues. Similar differences in gender appropriate topics of conversation amongst ethnic minorities are reported elsewhere (Grewal et al. 2005, Frey et al. 2013) and can influence service use. The concept of attending health visitor led groups was especially at odds with the cultural reliance on extended families as a source of guidance, coupled with a strong reluctance to discuss 'private' matters with strangers. This contrasts with findings reported by Donetto et al. (2013) where the majority of service users found 
local child health and breast feeding clinics an especially useful addition to home health visiting services. Paying greater attention to the acceptability and accessibility of services to minority ethnic groups is important in order to improve health outcomes for the minority ethnic population (Szczepura 2005). One solution to this, for example, is the provision of female only community based groups.

It was consistently reported that health visitors did not fully grasp the importance of the extended family in South Asian culture and the concept that their families were operating as a whole. Elsewhere it has been reported that consensus among family members is an important aspect of decision making in Asian families (Bowman and Singer 2001, Grewal et al. 2005), however in this study, health visitors were sometimes perceived to be uncomfortable involving family members in consultations. According to Puzan (2003), nursing discourse has been based on entrenched patterns of cultural dominance, which is perhaps reflected here with regard to the invisibility of extended families. The same participants also described the service as being helpful, and so were able to differentiate this from the rest of the service they received. Participants who felt isolated because their family were far away responded most positively to the health visitor's input and reported feeling supported and understood.

The findings are in line with previous research on SA and other ethnic minority perceptions of HVSs. Extended families can provide important support, but when there is conflict in the family they can contribute to feelings of depression and isolation (Wittkowski et al. 2011, Chan \& Leng 2004). Although negative feelings and isolation are common with post-natal depression, this was found to be further complicated by cultural, religious and family related factors in a study of South Asian mothers (Wittkowski et al. 2011).

For participants with extended family present, the issue of conflicting advice between the extended family and the health visitor was a frequent area of tension. When advice concerned parenting alone, family advice took precedence, however when the advice was health related, the health visitor's advice was more often accepted. Whether this was to maintain family harmony or respecting the views of elders, which is a core belief held by many South Asian families (Segal 1991), is not known. It was also noted that while participants regularly attributed their parenting approach to culture 'back home', it was evident that a variety of approaches were used by participants from similar cultural backgrounds. Further research is needed to explore these finding in relation to how concepts of ethnicity evolve and how differences between family expectations and the HVS are negotiated. 


\section{Study limitations}

Participants were those already accessing the HVS and a different recruitment strategy would be required to access the views of more marginalised clients who do not access the HVS. It was also possible that colleagues unconsciously approached clients with whom they had already developed a good rapport.

The inclusion criteria had limitations in that participants had to speak conversational English due to the lack of interpreting resources, excluding a significant part of the South Asian population, and having implications on the generalizability of the study. It is possible that interviews undertaken in participants own language may have produced more detailed data.

\section{Recommendations for practice and research}

Health visitors need to consider that South Asian clients may have difficulty accessing services because of a lack of familiarity with how the service operates. In order to promote equity of access, health visitors should be proactive in asking clients about their language needs and preference for use of an interpreter.

Sensitivity to family dynamics and their importance when giving health and parenting advice is crucial for health visitors if they are to deliver an effective HVS which meets the needs of the South Asian population. This study has revealed that the HVS is at least in part using an ethnocentric approach particularly when imparting parenting advice. There is a need for education and training in cultural issues and communication with minority ethnic clients to address this finding, (Henderson et al. 2011).

While it is important to gain a better understanding of what constitutes culturally appropriate services for South Asian clients, this should not be at the expense of further stereotyping ethnic groups. HVS should continue to strive towards providing holistic care at an individual level, based on individual assessment (Engebretson et al. 2008, Donetto et al. 2013).

Further research involving interpreter services is required to explore the views of non-English speaking clients. Observations of clinic contact with South Asian parents as well as home visits would add to the findings of this study and could support the design of culturally sensitive services which ultimately reduce health inequalities. 


\section{Conclusion}

There is a limited evidence base to inform the development of a HVS that is culturally appropriate to the needs of clients from minority ethnic backgrounds. This study contributes to understanding how HVSs in one setting in England are perceived by South Asian clients. There is no doubt that clients valued one-to-one support from their health visitor and found it responsive, particularly when suffering emotional distress. In keeping with previous research, there was evidence of poor communication and ethnocentric tendencies in the service. This largely related to language barriers, a perceived lack of understanding of the importance of extended family, and tensions between advice given by health visitors and that provided by the family. A key finding was that participants were more likely to accept health advice from their health visitor and parenting advice from their family. This study therefore makes a contribution to theoretical understanding which has an implication for health visiting practice and other health care professionals caring for South Asian clients.

\section{Source of Funding}

This research was conducted as part of post graduate study with no source of funding.

\section{Conflict of Interest}

None declared

\section{Acknowledgements}

The authors would like to express their appreciation to the participants who shared their experiences for this study.

\section{References}

Alexander C., Edwards R., \& Temple B. (2004) Access to services with interpreters: user views. York: Joseph Rowntree Foundation.

Beck C. (1993) Qualitative research: the evaluation of its credibility, fittingness and auditability. Western Journal of Nursing Research, 15 (2): 263-266.

Bhopal R. (2004) Glossary of terms relating to ethnicity and race: for reflection and debate. Journal of Epidemiology \& Community health, 58: 441-445 
Bhopal R. (2007) Race, Ethnicity, and Health in Multicultural Societies, Oxford University Press, Oxford.

Bowman K. \& Singer P. (2001). Chinese seniors' perspectives on end-of-life decisions. Social Science and Medicine, 53, 455-464.

Bradby H. (2003) Describing Ethnicity in Health Research. Ethnicity \& Health, 8(1): 5-13.

Bradby H. (2010) Institutional racism in mental health services: the consequence of compromised conceptualisation. Sociological Research, 15 (3): 8

Chivotti R. \& Piran N. (2003) Rigour and Grounded Theory Research. Journal of Advanced Nursing. 44 (4): 427-435.

Clegg A. (2003) Older South Asian patient and carer perceptions of culturally sensitive care in a community hospital setting. Journal of Clinical Nursing. 12 : 283-290.

Cowley S., Whittaker K., Grigulis A., Malone M, Donetto S., Wood H., Morrow E., \& Maben J. (2013) Why Health Visiting? A review of the literature about key health visitor interventions, processes and outcomes for children and families. London: National Nursing Research Unit, King's College London.

Culley L. (2006) Transcending transculturalism? Race, ethnicity and health-care. Nursing Inquiry 13 (2): 144-153

Department of Health (2007) Human Rights in Healthcare - A Framework for Local Action. The Stationery Office, London.

Department of Health (2010) Fair society, Healthy lives: The Marmot Review, The Stationery Office, London.

Department of Health (2011) Health Visitor Implementation Plan: A Call to Action, The Stationery Office, London.

Donetto S., Malone M., Hughes J., Morrow E., Cowley S. \& Maben J. (2013) Health visiting: the voice of service users - Learning from service users' experiences to inform the development of UK health visiting practice and services. London: National Nursing Research Unit, King's College London.

Douglas N. (2012) Befriending Breastfeeding: a home based antenatal pilot for South Asian families. Community practitioner 85 (6): 28 -31. 
Engebretson J. Mahoney J. \& Carlson E. (2008) Cultural competence in the era of evidencebased practice. Journal of Professional Nursing, 24 (3): 172-178.

Frey R., Gott M., Raphael D., Black S., Teleo-Hope L., Lee H. \& Wang Z. (2013) Where do I go from here? A cultural perspective on challenges to the use of hospice services. Health and Social Care in the Community (2013) 21(5), 519-529.

Garrett P., Dickson H., Whelan A. \& Roberto-Forero. (2008) What do non-English speaking patients value in acute care? Cultural competency from the patient's perspective: a qualitative study. Ethnicity \& Health 13 (5): 479-496.

Great Britain. Parliament. Equality Act 2010: Elizabeth II (2010) London: Stationery Office.

Green G., Bradby H., Chan A., \& Lee M. (2006) 'We are not completely westernised': Dual medical systems and pathways to health care among Chinese migrant workers in England. Social Science and Medicine 62 (6): 1498-1509

Grewal S., Bottorff J. \& Hilton B. (2005) The Influence of Family on Immigrant South Asian Women's Health. Journal of Family Nursing 11, 242-263.

Henderson S., Kendall E., \& See L. (2011) The effectiveness of culturally appropriate interventions to manage or prevent chronic disease in culturally and linguistically diverse communities: a systematic literature review. Health and Social Care in the Community 19(3), $225-24$.

Knight Jackson A. (2007) Cultural competence in health visiting practice: a baseline survey. Community Practitioner 80 (2): 17-22.

Leishman J. (2004) Perspectives of cultural competence in health care. Nursing Standard 19 (11): $33-38$.

Liu L., Davidson E., Bhopal R., et al. (2012) Adapting health promotion interventions to meet the needs of ethnic minority groups: mixed-methods evidence synthesis. Health Technology Assessments 16 (44). Available online at: http://www.journalslibrary.nihr.ac.uk/hta/volume-16/issue-44. Accessed 30/10/2014.

Office for National Statistics (2013). Detailed Characteristics for England and Wales, March 2011. The National Archives, London. 
O’Donell C., Higgins M., Cauhan R. \& Mullen K. (2007) “They think we're OK and we're not". A qualitative study of asylum seekers' access, knowledge and views to health care in the UK. BMC Health Services Research 7 (75). Available online at: http://www.biomedcentral.com/1472-6963/7/75. Accessed 21/01/2012.

Puzan E. (2003) The Unbearable whiteness of being (in nursing). Nursing Inquiry 10 (3): 193-200.

Szczepura A. (2005) Access to health care for ethnic minority populations. Ethnic Review 81: 141-147.

Segal U. (1991) Cultural variables in Asian Indian families. Families in Society, 72: 233-241.

Shadmi E. et al. (2010) Cancer care at the hospital-community interface: perspectives of patients from different cultural and ethnic groups. Patient Education and Counselling 79: 106-111.

Spence D. (2001) Prejudice, Paradox, and Possibility: Nursing People from Cultures Other Than One's Own. Journal of Transcultural Nursing 12 (2):100-106.

Starr S. \& Wallace D. (2011) Client perceptions of cultural competence of community based nurses. Journal of Community Health Nursing 28 (2): 57-69.

Strauss A. \& Corbin J. (1998) Basics of Qualitative Research. Techniques and Procedures for Developing Grounded Theory, $2^{\text {nd }}$ edition. Sage, California.

Suurmond J., Seeleman C., Rupp I., Goosen S., \& Stronks K. (2010) Cultural competence among nurse practitioners working with asylum seekers. Nurse Education Today, 30: 821826

Vydelingum V. (2000) South Asian patients' lived experience of acute care in an English Hospital: A phenomenological study. Journal of Advanced Nursing 32 (1): 100-107.

Vydelingum V. (2006) Nurses' experience of caring for South Asian minority ethnic patients in a general hospital in England. Nursing Inquiry 13(1): 23-32.

Wittkowski A., Zumla A., Glendenning S. \& FOX J.R.E. (2011) The experience of postnatal depression in South Asian mothers living in Great Britain: a qualitative study. Journal of Reproductive and Infant Psychology 29 (5): 480-492. 
Wohland P., Rees P., Norman P., Boden P. \& Jasinska M. (2010) Ethnic Population Projections for the UK \& Local Areas 2001-2051, Working Paper 10/02 [online] University of

Leeds,

Leeds.

Available

from:

http://www.geog.leeds.ac.uk/research/projects/migrants.html [Accessed 23 Jan 2012].

Yu J. (2011) A systematic review of issues around antenatal screening and prenatal diagnostic testing for genetic disorders: women of Asian origin in western countries. Health and Social Care in the Community (2012) 20(4), 329-346.

Table 1. Characteristics of participants

\begin{tabular}{|c|c|c|c|c|c|c|c|}
\hline Participant & $\begin{array}{c}\text { Country } \\
\text { of Origin }\end{array}$ & $\begin{array}{c}\text { Years in } \\
\text { education }\end{array}$ & $\begin{array}{c}\text { Live } \\
\text { with } \\
\text { extended } \\
\text { family }\end{array}$ & $\begin{array}{c}\text { Extended } \\
\text { family in } \\
\text { UK }\end{array}$ & $\begin{array}{c}\text { Extended } \\
\text { family } \\
\text { overseas }\end{array}$ & Religion & $\begin{array}{c}\text { First } \\
\text { time } \\
\text { mother }\end{array}$ \\
\hline 1 & Bangladesh & 9 & & yes & & Islam & yes \\
\hline 2 & Bangladesh & 16 & & & yes & Islam & no \\
\hline 3 & Pakistan & 11 & & yes & & Islam & yes \\
\hline 4 & India & 15 & & yes & & Hindu & no \\
\hline 5 & Pakistan & 16 & & yes & & Islam & yes \\
\hline 6 & India & 9 & & & yes & Islam & no \\
\hline 7 & India & 12 & yes & & & Hindu & yes \\
\hline 8 & India & 10 & yes & & & Hindu & yes \\
\hline 9 & India & 15 & & & yes & Hindu & Yes \\
\hline 10 & India & 11 & & & yes & Christian & no \\
\hline 11 & Pakistan & 9 & yes & & & Islam & no \\
\hline 12 & India & 12 & & & yes & Hindu & no \\
\hline 13 & Bangladesh & 10 & & Yes & & Islam & no \\
\hline 14 & Pakistan & 9 & yes & & Islam & yes \\
\hline 15 & Bangladesh & 10 & yes & & & Islam & no \\
\hline
\end{tabular}

